\title{
TUBERCULOSIS Y SALUD PÚBLICA: ¿DERECHOS INDIVIDUALES O DERECHOS COLECTIVOS?
}

\author{
Luis F. Llanos-Zavalaga ${ }^{1, a}$; José E. Velásquez-Hurtado²,b; \\ Patricia J. García ${ }^{1, b, c}$; Eduardo Gottuzzo $0^{2,3, c}$
}

\begin{abstract}
RESUMEN
La tuberculosis (TB) persiste como un problema de salud pública de grandes dimensiones en el Perú. La aparición de cepas fármaco-resistentes ha dificultado su control y puesto en cuestionamiento las medidas que actualmente se toman para la prevención y control. Un análisis desde los "determinantes sociales" relacionados con TB, confluyen hacia un tratamiento irregular, lo que ocasiona su persistencia y desarrollo de fármaco-resistencia. El objetivo es identificar el rol del Estado en el tratamiento de pacientes con TB; reconocer las dificultades del paciente en el cumplimiento del tratamiento, lo cual repercute en la salud colectiva; y discutir sus alternativas de manejo, basados en los derechos del paciente y la sociedad. La literatura internacional muestra experiencias límite entre los derechos individuales y colectivos, pero respaldado por políticas sanitarias y su legislación. En el Perú se requiere una nueva mirada que garantice la salud de la población sin vulnerar los derechos individuales.
\end{abstract}

Palabras clave: Tuberculosis; Salud pública; Resistencia a medicamentos; Derechos humanos (fuente: DeCS BIREME).

\section{TUBERCULOSIS AND PUBLIC HEALTH: ¿INDIVIDUAL RIGHTS OR COLLECTIVE RIGHTS?}

\begin{abstract}
Tuberculosis (TB) persists as a major public health problem in our country. The appearance of resistant strains has complicated its control and questioned the appropriateness of the current measures towards prevention and control. An analysis from social determinants related to TB, converge on irregular treatment that generates disease persistence and appearance of resistance to TB drugs. The objective of this paper is to identify the role of the government in the treatment of TB patients, to recognize difficulties of treatment adherence considering that its fulfillment depends on the patient, despite that it has direct consequences on public health, and to discuss TB management alternatives with an approach based on individual and collective human rights. International literature shows limit experiences of individual rights and collective ones, but based on health policies and health legislation. In Peru, a new approach is required to guarantee population health without infringing individual rights.
\end{abstract}

Key words: Tuberculosis; Public health; Drug resistance; Human rights (source: MeSH NLM).

\section{INTRODUCCIÓN}

La tuberculosis (TB) es una enfermedad transmitida por vía aérea que afecta en cualquier edad pero con mayor repercusión en la población económicamente activa. En el mundo, durante el 2010, se informó 8,8 millones de nuevos casos de TB; entre los que tenían coinfección con $\mathrm{VIH}$ se produjo 0,35 millones de muertes; y entre los que eran $\mathrm{VIH}$-negativos se produjo 1,1 millones de muertes ${ }^{(1)}$

En el Perú, en los últimos años, hay una disminución discreta de los casos de TB pansensible, y un incremento de los casos de TB multidrogorresistente (TB-MDR), excepto entre el 2009 al 2011. Alrededor del $90 \%$ de los casos de TB extremadamente resistente (TB-XDR) se encuentran en Lima y Callao. La TB pansensible predomina en la población de $20-59$ años $(66,05 \%$ de casos en 2011) y con mayor tasa de incidencia en los departamentos de la selva (Loreto, Ucayali y Madre de Dios) y Lima-Callao; en el 2010 la tasa de curación en la cohorte de esquema uno fue $89,5 \%$, menor al $90,0 \%$ que se informó en el periodo 2008-2010. En el caso de TB-MDR la tasa de curación en la cohorte del 2007 fue $66,0 \%$, con tasas de abandono mayores al 10,0\%.

\footnotetext{
Facultad de Salud Pública y Administración. Universidad Peruana Cayetano Heredia. Lima, Perú.

Facultad de Medicina Alberto Hurtado, Universidad Peruana Cayetano Heredia. Lima, Perú.

Instituto de Medicina Tropical Alexander von Humboldt, Universidad Peruana Cayetano Heredia. Lima, Perú.

Médico magister en Políticas, Planificación y Financiamiento en Salud; ${ }^{b}$ médico magister en Salud Pública; ${ }^{c}$ médico especialista en Enfermedades Infecciosas y Tropicales
} 
La base para el tratamiento de TB es la estrategia de tratamiento acortado estrictamente supervisado (Directly Observed Therapy Short-Course; DOTS). Pese a que el Perú fue un modelo mundial de su implementación ${ }^{(2)}$ la TB sigue en ascenso y con una situación más grave por el número creciente de casos de TB-MDR ${ }^{(3)}$. Algunas razones para este incremento son las demoras en los procesos de diagnóstico y tratamiento; la falta de continuidad en los tratamientos; las limitadas facilidades para los pacientes; y el manejo inadecuado de los efectos adversos.

Gracias a los avances, sin los cuales la situación hubiese sido más complicada, se conoce la génesis y control de la TB-MDR; se cuenta con una Unidad Técnica de Control de TB que vigila la situación epidemiológica y trabaja en la implementación y supervisión de las guías nacionales de manejo de TB/TB-MDR; existen comités competentes en la asignación de tratamientos TB-MDR a nivel nacional y regional; hay mayores facilidades para el diagnóstico mediante la introducción de pruebas rápidas de sensibilidad (MODS y Griess); y mayor capacidad económica para el desarrollo de actividades de capacitación y supervisión.

Los esquemas de tratamiento, efectivos para el manejo del caso individual, tienen limitaciones dentro de un Programa de Salud Pública. Existen factores asociados al individuo (ej. adherencia al tratamiento, efectos adversos) y de organización del Programa de Salud Pública (ej. tiempos diagnósticos, inicio del tratamiento, disponibilidad y continuidad del tratamiento, etc.) que pueden limitar su efectividad, conllevando al desarrollo de resistencia. La estrategia DOTS tiene un fundamento social expresado en el compromiso político y el tratamiento supervisado, pero al no enfrentarse problemas fundamentales como la pobreza e inequidad, desnutrición, hacinamiento y vivienda, discriminación y exclusión social, empleo, educación, limitaciones de los servicios de salud y seguridad social, resulta en un esfuerzo insuficiente.

\section{INTERVENCIÓN GUBERNAMENTAL SOBRE TB}

Ante la duda ¿es suficiente el riesgo que representa la TB en la salud pública del país, para proveer las bases éticas y legales que justifiquen la intervención del gobierno sobre el cuidado de la salud individual? Debemos plantear lo siguiente:

El fundamento ético para establecer programas de salud pública es reducir el riesgo de transmisión y limitar la amenaza de las enfermedades en la población en general. La teoría ética y la ética biomédica colocan especial énfasis en los derechos del individuo y en los principios de autonomía y autodeterminación. Estos principios éticos son tan importantes como su limitación en determinadas circunstancias.

Como señala Stuart Mill: El único propósito por el cual el poder puede ser ejercido sobre cualquier miembro de una comunidad civilizada, contra su voluntad, es para prevenir el daño a otros. Su propio bien, tanto físico y moral, no es suficiente garantía ${ }^{(4)}$. Los Principios de Siracusa ${ }^{(5)}$ señalan que: Toda vez que el concepto de moralidad pública varía en el tiempo y de una cultura a otra, el estado que invoque la moralidad pública como objetivo para limitar los derechos humanos, (...) deberá demostrar que la limitación de que se trate es esencial para mantener el respeto de los valores fundamentales de la comunidad. Por ello, cuando el ejercicio de la libertad individual puede ocasionar daño a otras, el estado debe intervenir, y allí no hay controversia. El principio de "evitar hacer daño" brinda una justificación para imponer limitaciones a la autonomía.

En algunos países la autoridad sanitaria puede limitar la libertad individual cuando una enfermedad constituye una amenaza poblacional, siendo reconocido por jurisprudencia constitucional. Hace más de cien años, la Corte Suprema en los Estados Unidos (EE. UU.) sostuvo que la constitución permitía a los estados promulgar: Las regulaciones razonables para proteger la salud pública y la seguridad pública; por el tiempo suficiente sin que: Contravengan la constitución de los EE. UU. ni infrinjan cualquier derecho garantizado o asegurado por este instrumento ${ }^{(6)}$. En el Perú, la responsabilidad social y de daño a terceros en salud pública aún requiere ser desarrollada.

Para la mayoría de las personas la salud es un tema personal. Sin embargo, asegurar la salud poblacional va más allá de la salud individual; es necesario incluir políticas sanitarias nacionales, independientemente del desarrollo económico (7), involucrando a actores sociales (pacientes y sus familias, organizaciones de la sociedad civil incluidas las de pacientes, trabajadores de salud y los órganos de gobierno en sus diferentes niveles).

Desde el campo de los derechos humanos, la práctica de la Salud Pública implica discrepancias entre los llamados derechos humanos individuales y los derechos humanos colectivos de la población en su conjunto ${ }^{(8)}$. Los Principios de Siracusa señalan que: La salud pública puede invocarse como motivo para limitar ciertos derechos a fin de permitir a un Estado adoptar medidas para hacer frente a una grave amenaza a la salud de la población o de alguno de sus miembros ${ }^{(5)}$. 
La ética en Salud Pública está focalizada en el diseño e implementación de medidas para vigilar y mejorar la salud poblacional. Además, considera condiciones estructurales que promueven o inhiben el desarrollo de comunidades saludables y no solo el cuidado de la salud. Considera los siguientes elementos clave: (1) Disparidades en el estado de salud, acceso a atención de salud y a beneficios de la investigación médica; (2) Respuesta a la amenaza de enfermedades infecciosas; (3) Cooperación internacional en monitoreo y vigilancia sanitaria; (4) Explotación de personas en países de bajos ingresos; (5) Promoción de la salud; y (6) Participación, transparencia y rendición de cuentas (accountability). La respuesta a las enfermedades infecciosas incluye esfuerzos para contener su transmisión; ello genera preguntas complejas relacionadas como si es necesario recortar las libertades individuales o cuán apropiado es hacerlo en salvaguarda del bienestar de otras personas ${ }^{(9)}$.

Upshur ${ }^{(10)}$ considera cuatro principios que justifican intervenciones en salud pública por sobre el derecho individual: (1) El principio de daño; el único propósito para ejercer el poder contra cualquier miembro de la sociedad contra su voluntad es para prevenir el daño a los demás. (2) El principio del medio menos restrictivo; reconoce que se debe usar el medio menos restrictivo de las libertades individuales, y que la fuerza y autoridad del estado solo debe emplearse en circunstancias excepcionales. (3) El principio de reciprocidad; señala que garantizadas las acciones de salud pública existe la obligación de una entidad social para apoyar a la persona o comunidad en descargar sus responsabilidades éticas, pudiendo involucrar mayores costos y tiempos que deben ser compensados.

(4) El principio de transparencia; está relacionado con el contexto y la toma de decisiones. Para ello los stakeholders deben estar involucrados en el proceso para tomar decisiones clara, responsable, y libre de interferencia y coerción.

\section{TB COMO UN PROBLEMA DE SALUD PÚBLICA; MÁS ALLÁ DE LOS DERECHOS INDIVIDUALES}

Existe tensión entre los derechos individuales de las personas y las necesidades en salud pública (necesidades colectivas) para el control de las enfermedades infecciosas ${ }^{(11)}$.

Este debate en el caso de TB incluye diversos momentos de la enfermedad: (1) Tamizaje para descubrir la presencia de enfermedad; (2) Vigilancia y reporte, a los registros de salud pública, de las personas enfermas; (3)
Seguimiento de contactos; (4) Tratamiento obligatorio de los casos infectados; y (5) La cuarentena o aislamiento de aquellos en quienes su condición biológica o su comportamiento representa una amenaza a la salud pública. También vincula otros aspectos controversiales como: la confidencialidad diagnóstica; la obligatoriedad de pruebas diagnósticas; la declaración obligatoria del paciente para seguimiento sanitario; la imposición de tratamientos; el internamiento o medidas extraordinarias; el consejo sexual y posibles medidas coactivas de la vida privada; la creación de centros especiales de asistencia; entre otros.

Para Bayer (12), algunas interrogantes específicas, éticas y legales, rodean al control de la tuberculosis con énfasis particular en las libertades civiles de cuatro estrategias: (1) Provisión de servicios de apoyo para motivar a completar el tratamiento; (2) La terapia directamente observada (DOTS) para vigilar la adherencia al tratamiento; (3) La detención involuntaria de pacientes no adherentes; y (4) La administración forzada de medicación antituberculosa. A continuación describiremos algunas reflexiones existentes sobre tamizaje, tratamiento y aislamiento.

¿Para proteger a una población vulnerable, qué elementos de una intervención efectiva de salud pública pueden justificar la invasión de la integridad corporal y privacidad, y deben estar presentes en programas de tamizaje obligatorios? En general, se promueve que las personas reconozcan cuáles son los síntomas asociados a TB, y que acudan de manera voluntaria a hacerse el despistaje con prueba de esputo y radiografía de tórax. Sin embargo, en TB se acepta una trasgresión de principios éticos y legales en el tamizaje obligatorio para identificar infección con TB latente en los migrantes de países del hemisferio sur a los países desarrollados del hemisferio norte, y posterior tratamiento de los casos positivos. En cierta forma se ofrece al individuo la posibilidad de un diagnóstico y tratamiento, aunque se debe reconocer que en muchos de sus países de origen aún se ofrece vacunación con BCG (lo que podría condicionar un PPD positivo), que la infección solo representa un riesgo para otros cuando progresa a una enfermedad activa y que solo $10 \%$ de los casos con TB latente desarrollan la enfermedad.

Gostin (13) abre reflexiones éticas, al señalar que: La legislación en salud pública se ha enfocado principalmente en las infecciones presentes, [pero] no existe razón para limitar la doctrina de amenaza directa en esta dirección, implicando el considerar infecciones potenciales. Ello abre posibilidades a actividades que puedan violar principios éticos en nombre de la salud 
pública por infecciones potenciales, basados en el criterio "razonablemente previsible".

Otro elemento es: ¿quiénes deben someterse al tamizaje obligatorio? Este tamizaje debe ser inclusivo para todos quienes presenten un perfil epidemiológico de riesgo. Se debe encontrar un balance entre las siguientes características: necesidad, razonabilidad, efectividad, proporcionalidad, justicia distributiva -discriminación contra un grupo social (ej. inmigrantes) y transparencia.

La estrategia impulsada por la OPS/OMS es el tratamiento directamente observado (DOTS), por el riesgo de no curación; desarrollo de resistencia; transmisión de la enfermedad o muerte, asociadas a pobre adherencia al tratamiento, lo complicado del tratamiento y la frecuencia de eventos adversos. La pobre adherencia al tratamiento es común en tratamientos crónicos; sin embargo, las fallas en completar tratamiento en otras enfermedades, no tienen las mismas consecuencias negativas, individuales y de salud pública. Existe un consenso universal que la TB representa una amenaza global en salud pública y que se requiere implementar la estrategia DOTS para todos los casos hasta su curación.

El DOTS universal ha provocado oposición en determinadas sociedades. Se ha argumentado que dicho esfuerzo acarreará una enorme pérdida de los escasos recursos. Los fondos que podrían ser mejor usados en quienes tienen más necesidad serían desviados a la supervisión de quienes serán adherentes por sí mismos. También se ha considerado como una intrusión no ética sobre la autonomía, como una "molestia gratuita", como una violación del requerimiento constitucional por la alternativa menos restrictiva.

En el caso de los EE. UU. la ley concedió bastante amplitud a las autoridades de salud pública para enfocarse en la necesidad de identificar, tratar y limitar la actividad de quienes sean infecciosos. En 1992 los Centros de Control de Enfermedades (CDC) de Atlanta desarrollaron una amplia revisión de las leyes de los estados en control de TB encontrando que 43 estados proveen cuarentena para los pacientes con TB dentro de sus propias casas, 35 especifican que esta debe durar hasta que no sea contagioso ${ }^{(14)}$.

Una aproximación efectiva para mejorar la adherencia al tratamiento debe ser sensible a los obstáculos que afrontan las personas socialmente marginadas y económicamente vulnerables para acceder al tratamiento, como las personas sin hogar, drogadictos, alcohólicos, enfermos psiquiátricos, etc. Una persona no puede ser moralmente responsable por fallar en adherirse a estándares éticos y legales si es incapaz de hacerlo. El principio ético obliga a reconocer la eliminación de impedimentos que inciden en la capacidad de la persona para cooperar en su propio cuidado.

No se debe esperar que las personas razonablemente cumplan con su cuidado a menos que cuenten o se les provea de una residencia segura u otros apoyos sociales. Por ello, se deben realizar análisis económicos de iniciativas de apoyo social como casas de hospedaje, y apoyo alimentario luego de completar su tratamiento (como incentivo positivo a su cumplimiento); teniendo presente que las fallas al tratamiento implican transmisión de enfermedad y desarrollo potencial de drogorresistencia, ahondando el problema de salud pública.

Desde una perspectiva ética y práctica, las autoridades de salud pública reconocen que es preferible hacer esfuerzos positivos para motivar la adherencia voluntaria con precauciones de salud antes de recurrir a acciones coercitivas. Las autoridades de salud reconocen la importancia de proveer apoyo social y otros (pasajes, alimentos y pagos en efectivo para acudir al tratamiento), pero son pocas las experiencias con evaluaciones económicas.

Pese a ello persisten las siguientes preguntas ¿si una parte del gobierno falla al proveer un servicio particular (como asistencia social), se justifica imponer restricciones en las libertades individuales reconocidas constitucionalmente? ¿Puede la doctrina de la alternativa menos restrictiva, que inicialmente sirvió como una limitante negativa a las acciones de gobierno, imponer obligaciones positivas a los gobiernos, como una obligación de brindar apoyos sociales para facilitar la adherencia?

Los principios éticos, legales y constitucionales en países como los EE. UU. reconocen la autoridad del estado para confinar a las personas con enfermedades infecciosas por la amenaza que representan para otros. Este poder priva al individuo de su libertad en nombre de la salud pública y brinda a los funcionarios de salud pública de una autoridad que no se distingue del sistema criminal judicial, desde una perspectiva individual. El confinamiento hasta la cura puede ser considerado probablemente constitucional para pacientes infecciosos quienes no se adhieren al tratamiento, más aun en contextos de TB-MDR/TB-XDR.

En 1979 la Corte Suprema en Addington frente a Texas (15) señaló que: El confinamiento por cualquier propósito, constituye una privación de la libertad y 
requiere el debido proceso de protección. Más aun es irrefutable que el internamiento involuntario a un hospital mental puede ocasionar consecuencias sociales adversas al individuo, este estigma puede tener un impacto significante en el individuo. En West Virginia, en 1980, la Corte Suprema de Apelaciones señaló que la Ley de Control de Tuberculosis era inconstitucional porque no garantizaba el derecho a consejería sin proveer una confrontación ni testigos de manera clara y convincente, como la prueba estándar que requería la Corte Suprema ${ }^{(13)}$.

En Nueva York, en 1993, el departamento de salud adoptó regulaciones para el confinamiento de quienes se considere que no completarán el tratamiento antituberculoso: Una probabilidad sustancial de un comportamiento no confiable, basado en su comportamiento pasado y presente. Fue significativo en estas regulaciones imponer la cuarentena, incluso si no se han agotado todos los elementos de la aproximación menos restrictiva. No se requiere esperar a que el paciente falle en el conjunto de intervenciones ${ }^{(16)}$.

En la legislación que ha establecido el derecho a rehusar medicación se asume que los individuos pueden retener el derecho a cierta autodeterminación, incluso en condiciones de confinamiento, porque ellos representan un riesgo para ellos mismos u otros. Un paciente civilmente comprometido puede ser obligado a tomar la medicación solo después de una determinación judicial que demuestre que el individuo carece de capacidad para tomar una decisión razonada sobre un tratamiento particular.

Un paciente con capacidad de decidir puede escoger privarse de tratamiento incluso a riesgo de su muerte. Ello es lo fundamental del principio de consentimiento informado. Pero dichos pacientes no tienen derecho a poner en peligro a otros. No obstante, la paradoja persiste: el estado puede confinar a un individuo no adherente al tratamiento, pero no puede imponer tratamiento a un paciente confinado.

En Sudamérica existen experiencias en otras enfermedades; en Brasil existe un marco legal para entrar a los domicilios de viviendas cerradas para acciones de vigilancia y control en dengue (17). En el Perú la Ley Orgánica de Gobiernos Regionales y sus modificatorias establecen que es función específica de los gobiernos regionales formular, aprobar, ejecutar, evaluar, dirigir, controlar y administrar las políticas de salud de la región, en concordancia con las políticas nacionales y los planes sectoriales. Existe, por tanto, el marco legal nacional para proponer iniciativas frente al problema de TB/TB-MDR.

\section{¿QUÉ HACER EN EL CASO PERUANO?}

Este debate debe establecerse en el Perú frente al problema creciente de TB-MDR/TB-XDR. Se requiere la participación de los legítimos actores sociales en la construcción de los procesos. A continuación se describen algunos criterios para diversos momentos.

En el caso del tamizaje se requiere garantizar una evaluación (examen físico más baciloscopía de esputo) de todos los sintomáticos respiratorios y los contactos de pacientes con TB. Para los contactos de pacientes con TB pansensible con baciloscopía negativa se requerirá garantizar el cumplimiento de la quimioprofilaxis, priorizando a menores de 15 años y otras poblaciones vulnerables (como personas con enfermedades crónicas). El sistema de salud debe garantizar la identificación y evaluación activa periódica de los contactos de casos de TB pansensible. La realidad epidemiológica exige el compromiso ético de utilizar herramientas tecnológicas más modernas que permitan diagnósticos más temprano y oportunos.

En el caso de los contactos de pacientes con TBMDR/TB-XDR se requiere la evaluación de cada uno de los contactos y definir la periodicidad en la que se deberá realizar su despistaje. Se considera que dicha periodicidad deberá estar relacionada con su perfil epidemiológico local, y realizarse incluso posterior al alta del paciente con TB-MDR/TB-XDR.

En el caso del tratamiento de pacientes con TB,se requiere analizar las alternativas para garantizar el cumplimiento de su tratamiento. Una primera alternativa a agregarse sobre las existentes es que durante la consejería inicial que reciben los pacientes se les involucre activamente y se solicite la firma de un documento de carácter legal, con el cual el paciente se compromete a cumplir su tratamiento y a reconocer que podrá ser sujeto de confinamiento no voluntario en caso de que ello no ocurra. Este confinamiento podrá ser hasta que negativice su baciloscopía o hasta que complete su tratamiento con baciloscopía negativa. Simultáneamente, se deberán establecer los mecanismos y procedimientos legales necesarios para que ello no constituya una violación de los derechos humanos individuales.

Ello implica habilitar locales especializados donde los pacientes completen su tratamiento mediante una atención y seguimiento apropiado, con condiciones de bioseguridad adecuadas, coordinando con las autoridades regionales, locales y de los diferentes sectores. En el marco del principio de reciprocidad se deberán establecer los mecanismos para garantizar 
que los pacientes reciban alojamiento, alimentación y un estipendio que permita minimizar la carga de enfermedad sobre su familia.

Asimismo, el sistema deberá asegurar los mecanismos para evitar la falta de medicinas y analizar los diversos estímulos, considerando que en algunos casos pueden convertirse en perversos. Se conoce que los alimentos que reciben los pacientes con TB sirven como complemento en la alimentación familiar, y que algunos pacientes suspenden su medicación a fin de continuar recibiéndolos. También se deberán establecer mecanismos que en lugar de sancionar el incumplimiento permitan premiar el cumplimiento. Por ejemplo, que puedan recibir alimentos luego de culminar su tratamiento o sea dado de alta, o reciban un incentivo por acabar su tratamiento y haber negativizado.

En conclusión, en el Perú este tema debe ser abordado en el corto plazo. Los servicios de atención de pacientes con TB deben adecuarse constantemente, y se requiere de normatividades que garanticen la salud de la población sin vulnerar los derechos humanos de cada individuo. Desde la salud pública se requiere trabajar articuladamente con los gobiernos municipales y locales para mejorar la prestación y brindar servicios de apoyo social que permitan mejorar la adherencia en TB y TBMDR, pero deben también establecerse los mecanismos legales que permitan manejar las situaciones de no adherencia. Asimismo, esta reflexión ética debe dialogarse y enriquecerse con aportes de pacientes con TB/TB-MDR/TB-XDR y sus familiares, de la asociación de enfermos con TB, de funcionarios de salud del nivel local y nacional a fin de establecer acciones claras para reforzar la adherencia al tratamiento, en beneficio del individuo y de la sociedad en pleno.

\section{Fuentes de financiamiento}

Autofinanciado.

\section{Conflictos de interés}

Los autores declaran no tener conflictos de interés en la publicación del presente artículo.

\section{REFERENCIAS BIBLIOGRÁFICAS}

1. Organización Mundial de la Salud. Global Tuberculosis Control. WHO Report 2011. Ginebra: WHO; 2011.

2. Bonilla C. Situación de la tuberculosis en el Perú. Acta Med Per. 2008;25(3):163-70.
3. Llanos-Zavalaga LF, Mayca JA, Salazar D. Estudio de Línea de Base para el Proyecto "Haciendo la Diferencia: Consolidando una Respuesta Amplia e Integral a la Tuberculosis en el Perú, Octava Ronda del Fondo Mundial de Lucha contra el VIH/SIDA, Tuberculosis y Malaria". Informe Final. Lima: PARSALUD/MINSA; 2011.

4. Mill JS. On Liberty. Cambridge: Cambridge University Press; 1989.

5. University of Minesota. United Nations, Economic and Social Council, Siracusa Principles on the Limitation and Derogation Provisions in the International Covenant on Civil and Political Rights, U.N. Doc. E/CN.4/1985/4, Annex. Washington, DC: United Nations; 1985.

6. U.S. Supreme Court. Jacobson v. Massachusetts, 197 US II. Massachusetts: U.S. Supreme Court; 1905.

7. Málaga H. Salud Pública. Enfoque bioético. Caracas: Editorial Disinlimed CA; 2005.

8. Kass NE. An ethics framework for public health. Am J Public Health. 2001;91(11):1776-82.

9. Coleman $\mathbf{C H}$, Bouësseau MC, Reis A. The contribution of ethics to public health. Bull World Health Organ. 2008;86(8):578-A.

10. Upshur RE. Principles for the justification of public health intervention. Can J Public Health. 2002;93(2):101-3.

11. Ortiz Z. Las enfermedades de la pobreza desde la epidemiología. Médicos Hoy. Revista de la Confederación Médica de la República Argentina [Internet]; 2005 [consultado el 15 de octubre de 2011]. Disponible en: http:// epidemiologia.ar11.toservers.com/pdf/publicaciones_cie/2005/ enfermedades_Pobreza_desde_Epidemiologia_2005.pdf

12. Bayer R, Dupuis L. Tuberculosis, public health and civil liberties. Annu Rev Public Health. 1995;16:307-26.

13. Gostin L. The resurgent tuberculosis epidemic in the era of AIDS: reflections on public health, law and society. MD Law Rev. 1995;54(1):1-131.

14. Tuberculosis control laws--United States, 1993. Recommendations of the Advisory Council for the Elimination of Tuberculosis (ACET). MMWR Recomm Rep. 1993;42(RR-15):1-28.

15. U.S. Supreme Court. Addington v. Texas, 441 U.S. 418. Massachusetts: U.S. Supreme Court; 1979.

16. New York, NY, Health Code, Article I I. Section 11.47

17. Brasil, Ministério da Saúde. Programa Nacional de Controle da Dengue: Amparo Legal à Execução das Ações de Campo - Imóveis Fechados, Abandonados ou com Acesso não Permitido pelo Morador. 2da ed. Brasilia: Ministério da Saúde; 2006.

Correspondencia: Luis Fernando Llanos Zavalaga

Dirección: Calle Las Lilas 435, Lima 14, Perú.

Teléfono: (511) 989295094.

Correo electrónico: luis.Ilanos@upch.pe 